\title{
Molecular Architecture via Coordination: Self-Assembly of Nanoscale Hexagonal Metallodendrimers with Designed Building Blocks
}

\author{
Haibo Yang,${ }^{*}{ }^{\dagger}$ Neeladri Das,${ }^{\dagger}$ Feihe Huang, ${ }^{\dagger}$ Adam M. Hawkridge, ${ }^{\S}$ \\ David C. Muddiman, ${ }^{\S}$ and Peter J. Stang ${ }^{*}, \dagger$
}

Contribution from the Department of Chemistry, University of Utah, 315 South 1400 East, Rm, 2020, Salt Lake City, Utah, 84112 and W. M. Keck FT-ICR Mass Spectrometry Laboratory and Department of Biochemistry and Molecular Biology, Mayor Clinic and Foundation, Rochester, Minnesota 55905.

stang@ chem.utah.edu

\section{Table of Contents}

Experimental Section....................................................................... 2

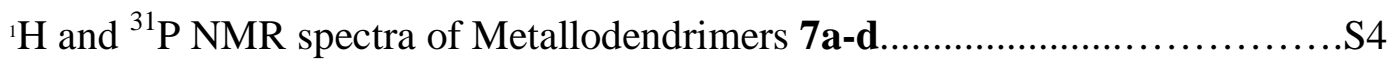

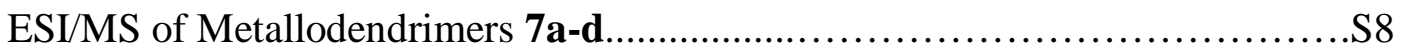

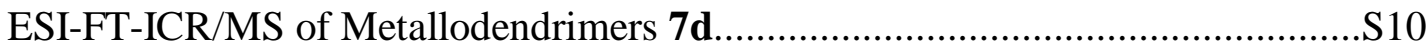

* To whom correspondence should be addressed.

$\dagger$ University of Utah.

$\S$ North Carolina State University 


\section{General Procedure for the Preparation of Hexagonal Metallodendrimers 7a-d}

Nitrate 6 and the appropriate [G0]-[G3] dendritic precursors (5a-d) were placed in a 2dram vial. $1 \mathrm{~mL}$ mixture solvent (Acetone- $d_{6} / \mathrm{D}_{2} \mathrm{O}: 10 / 1$ ) was added. The reaction mixture was stirred overnight at $55^{\circ} \mathrm{C}$. The solution was evaporated to dryness, and the product was collected.

[G0]-Metallodendrimer (7a). Yield: 96\%. ${ }^{1} \mathrm{H}$ NMR (Acetone- $d_{6} / \mathrm{D}_{2} \mathrm{O}: 10 / 1,300 \mathrm{MHz}$ ): $\delta 8.99$ (d, J=5.7 Hz, 12H, $\left.\mathrm{H}_{\alpha}-\mathrm{Py}\right), 7.89$ (d, J=6.0Hz, 12H, $\left.\mathrm{H}_{\beta}-\mathrm{Py}\right), 7.64$ (d, J=7.8Hz, 12H, $\left.\mathrm{H}_{\mathrm{a}}-\mathrm{Ph}\right), 7.50\left(\mathrm{~d}, J=7.2 \mathrm{~Hz}, 12 \mathrm{H}, \mathrm{H}_{\mathrm{b}} \mathrm{Ph}\right), 7.36-7.45(\mathrm{~m}, 24 \mathrm{H}, \operatorname{Ar} H$ and $\mathrm{Ph} H), 5.22(\mathrm{~s}, 6 \mathrm{H}$, $\left.\mathrm{OCH}_{2} \mathrm{Ph}\right), 1.40-1.41\left(\mathrm{~m}, 72 \mathrm{H}, \mathrm{PCH}_{2} \mathrm{CH}_{3}\right), 1.07-1.20\left(\mathrm{~m}, 108 \mathrm{H}, \mathrm{PCH}_{2} \mathrm{CH}_{3}\right) .{ }^{31} \mathrm{P}\left\{{ }^{1} \mathrm{H}\right\} \mathrm{NMR}$ (Acetone- $\left.d_{6} / \mathrm{D}_{2} \mathrm{O}: 10 / 1,121.4 \mathrm{MHz}\right): \delta 18.5\left(\mathrm{~s},{ }^{1} J_{\mathrm{Pt}-\mathrm{P}}=2646.0 \mathrm{~Hz}\right)$. Anal. Calcd for $\mathrm{C}_{192} \mathrm{H}_{258} \mathrm{~N}_{12} \mathrm{O}_{24} \mathrm{P}_{12} \mathrm{Pt}_{6} \mathrm{H}_{2} \mathrm{O}$ : C, 49.29; H, 5.60; N, 3.59. Found: C, 49.01; H, 5.89; N, 3.33.

[G1]-Metallodendrimer (7b). Yield: 98\%. ${ }^{1} \mathrm{H}$ NMR (Acetone- $d_{6} / \mathrm{D}_{2} \mathrm{O}: 10 / 1,300 \mathrm{MHz}$ ): $\delta 8.95\left(\mathrm{~d}, J=5.4 \mathrm{~Hz}, 12 \mathrm{H}, \mathrm{H}_{\alpha}-\mathrm{Py}\right), 7.86\left(\mathrm{~d}, J=5.4 \mathrm{~Hz}, 12 \mathrm{H}, \mathrm{H}_{\beta}-\mathrm{Py}\right), 7.62$ (d, J=8.1Hz, 12H, $\left.\mathrm{H}_{\mathrm{a}}-\mathrm{Ph}\right), 7.48$ (d, J=8.9Hz, 12H, $\left.\mathrm{H}_{\mathrm{b}}-\mathrm{Ph}\right), 7.26-7.39(\mathrm{~m}, 39 \mathrm{H}, \mathrm{Ar} H$ and $\mathrm{Ph} H), 6.69(\mathrm{~d}$, $J=1.5 \mathrm{~Hz}, 6 \mathrm{H}, \mathrm{ArH}), 6.59$ (t, 3H, $\mathrm{ArH}), 5.16\left(\mathrm{~s}, 6 \mathrm{H}, \mathrm{OCH}_{2} \mathrm{Ar}\right), 5.06$ (s, 12H, OCH $2 \mathrm{Ph}$ ), $1.37\left(\mathrm{~m}, 72 \mathrm{H}, \mathrm{PCH}_{2} \mathrm{CH}_{3}\right), 1.07-1.18\left(\mathrm{~m}, 108 \mathrm{H}, \mathrm{PCH}_{2} \mathrm{CH}_{3}\right) .{ }^{31} \mathrm{P}\left\{{ }^{1} \mathrm{H}\right\}$ NMR (Acetone- $d_{6} /$ $\left.\mathrm{D}_{2} \mathrm{O}: 10 / 1,121.4 \mathrm{MHz}\right): \delta 18.5\left(\mathrm{~s},{ }^{1} J_{\mathrm{Pt}-\mathrm{P}}=2644.8 \mathrm{~Hz}\right)$. Anal. Calcd for $\mathrm{C}_{234} \mathrm{H}_{294} \mathrm{~N}_{12} \mathrm{O}_{30} \mathrm{P}_{12} \mathrm{Pt}_{6}:$ C, 53.06; H, 5.59; N, 3.17. Found: C, 53.30; H, 5.97; N, 2.82.

[G2]-Metallodendrimer (7c). Yield: 99\%. ${ }^{1} \mathrm{H}$ NMR (Acetone- $d_{6} / \mathrm{D}_{2} \mathrm{O}: 10 / 1,300 \mathrm{MHz}$ ): $\delta 8.99\left(\mathrm{~d}, J=5.1 \mathrm{~Hz}, 12 \mathrm{H}, \mathrm{H}_{\alpha}-\mathrm{Py}\right), 7.87$ (d, J=5.4Hz, 12H, $\left.\mathrm{H}_{\beta}-\mathrm{Py}\right), 7.64$ (d, J=7.8Hz, 12H, $\left.\mathrm{H}_{\mathrm{a}} \mathrm{Ph}\right), 7.51\left(\mathrm{~d}, J=8.1 \mathrm{~Hz}, 12 \mathrm{H}, \mathrm{H}_{\mathrm{b}}-\mathrm{Ph}\right), 7.27-7.41(\mathrm{~m}, 69 \mathrm{H}, \mathrm{Ar} H$ and $\mathrm{Ph} H), 6.69(\mathrm{~m}, 18 \mathrm{H}$, $\mathrm{ArH}), 6.58(\mathrm{~m}, 9 \mathrm{H}, \mathrm{ArH}), 5.18\left(\mathrm{~s}, 6 \mathrm{H}, \mathrm{OCH}_{2} \mathrm{Ar}\right), 5.05$ (br, 36H, OCH $\mathrm{OC}_{2} \mathrm{Ar}$ and $\left.\mathrm{OCH}_{2} \mathrm{Ph}\right)$, $1.40\left(\mathrm{~m}, 72 \mathrm{H}, \mathrm{PCH}_{2} \mathrm{CH}_{3}\right), 1.09-1.17\left(\mathrm{~m}, 108 \mathrm{H}, \mathrm{PCH}_{2} \mathrm{CH}_{3}\right) .{ }^{31} \mathrm{P}\left\{{ }^{1} \mathrm{H}\right\}$ NMR (Acetone- $d_{6} /$ $\left.\mathrm{D}_{2} \mathrm{O}: 10 / 1, \quad 121.4 \mathrm{MHz}\right): \delta 18.5\left(\mathrm{~s},{ }^{1} J_{\mathrm{Pt}-\mathrm{P}}=2645.5 \mathrm{~Hz}\right)$. Anal. Calcd for $\mathrm{C}_{318} \mathrm{H}_{366} \mathrm{~N}_{12} \mathrm{O}_{42} \mathrm{P}_{12} \mathrm{Pt}_{6}: \mathrm{C}, 58.13 ; \mathrm{H}, 5.61 ; \mathrm{N}, 2.56$. Found: C, 57.99; H, 5.86; N, 2.32. 
[G3]-Metallodendrimer (7d). Yield: 98\%. ${ }^{1} \mathrm{H}$ NMR (Acetone- $d_{6} / \mathrm{D}_{2} \mathrm{O}: 10 / 1,300 \mathrm{MHz}$ ): $\delta 8.92$ (br, 12H, $\left.\mathrm{H}_{\alpha}-\mathrm{Py}\right), 7.81$ (br, 12H, $\left.\mathrm{H}_{\beta}-\mathrm{Py}\right), 7.59$ (br, $12 \mathrm{H}, \mathrm{H}_{\mathrm{a}}-\mathrm{Ph}$ ), 7.49 (d, J=7.8Hz, 12H, $\left.\mathrm{H}_{\mathrm{b}}-\mathrm{Ph}\right), 7.24-7.31$ (m, 69H, $\mathrm{Ar} H$ and $\left.\mathrm{Ph} H\right), 6.62$ (br, 42H, $\left.\mathrm{ArH}\right), 6.51$ (br, 21, $\mathrm{Ar} H$ ), 4.95 (br, $90 \mathrm{H}, \mathrm{OCH}_{2} \mathrm{Ar}$ and $\left.\mathrm{OCH}_{2} \mathrm{Ph}\right), 1.35\left(\mathrm{~m}, 72 \mathrm{H}, \mathrm{PCH}_{2} \mathrm{CH}_{3}\right), 1.04-1.09(\mathrm{~m}, 108 \mathrm{H}$, $\left.\mathrm{PCH}_{2} \mathrm{CH}_{3}\right) .{ }^{31} \mathrm{P}\left\{{ }^{1} \mathrm{H}\right\}$ NMR (Acetone- $\left.d_{6} / \mathrm{D}_{2} \mathrm{O}: 10 / 1,121.4 \mathrm{MHz}\right): \delta 18.5\left(\mathrm{~s},{ }^{1} J_{\mathrm{Pt}-\mathrm{P}}=\right.$ $2646.3 \mathrm{~Hz}$ ). Anal. Calcd for $\mathrm{C}_{486} \mathrm{H}_{510} \mathrm{~N}_{12} \mathrm{O}_{66} \mathrm{P}_{12} \mathrm{Pt}_{6}: \mathrm{C}, 64.02 ; \mathrm{H}, 5.64 ; \mathrm{N}, 1.84$. Found: $\mathrm{C}$, $63.73 ; \mathrm{H}, 5.76 ; \mathrm{N}, 1.71$. 
Figure S1. A) ' $\mathrm{H}$ and B) ${ }^{31} \mathrm{P}$ NMR spectra of $7 \mathbf{a}$ in Acetone- $d_{6} / \mathrm{D}_{2} \mathrm{O}(10 / 1)$

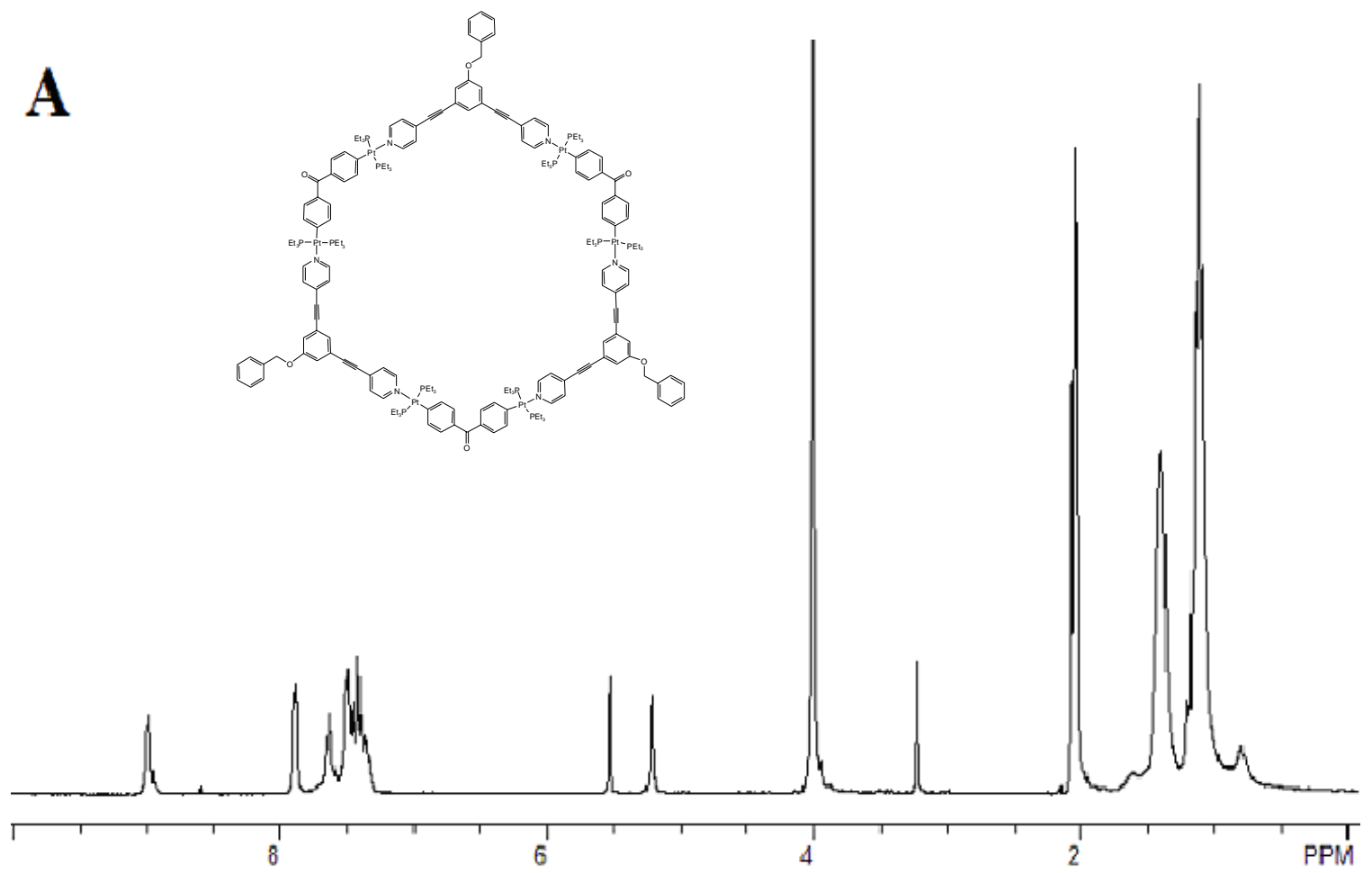

B

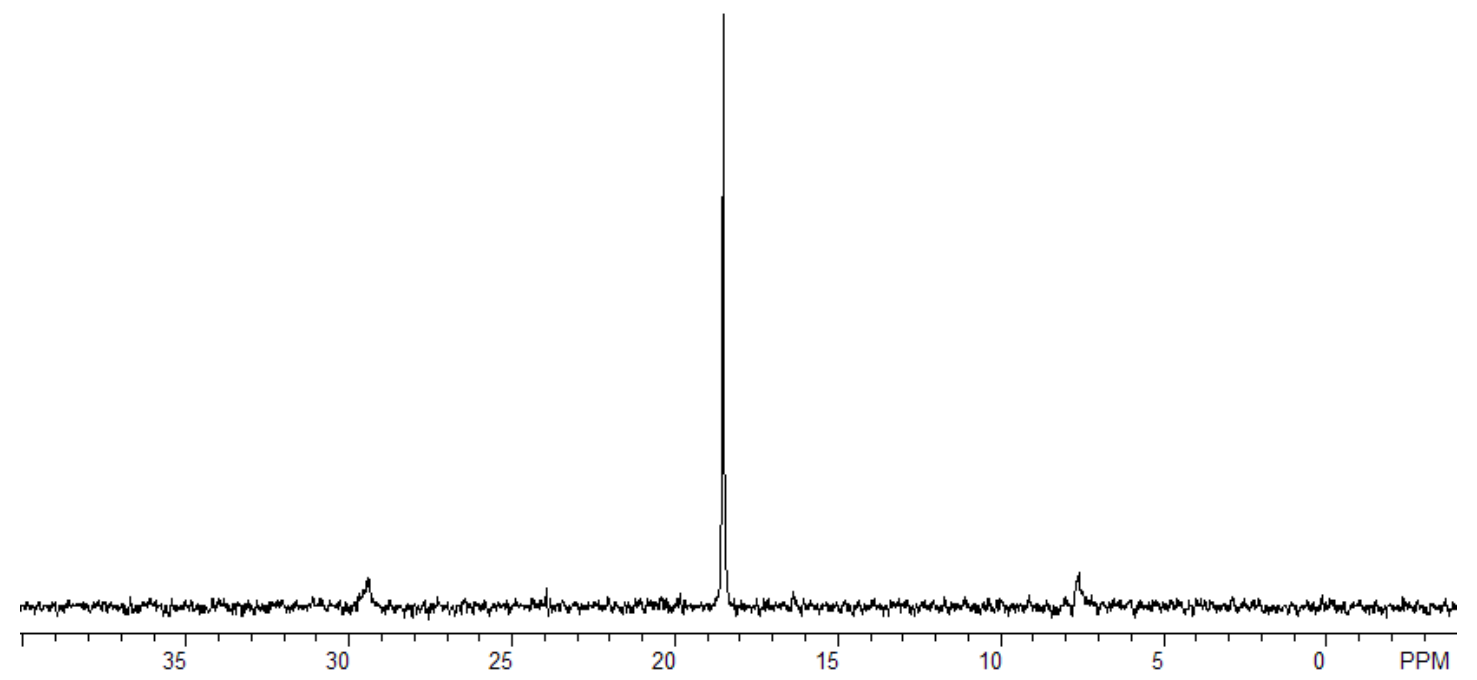


Figure S2. A) ' $\mathrm{H}$ and B) ${ }^{31} \mathrm{P}$ NMR spectra of $7 \mathbf{b}$ in Acetone- $d_{6} / \mathrm{D}_{2} \mathrm{O}(10 / 1)$

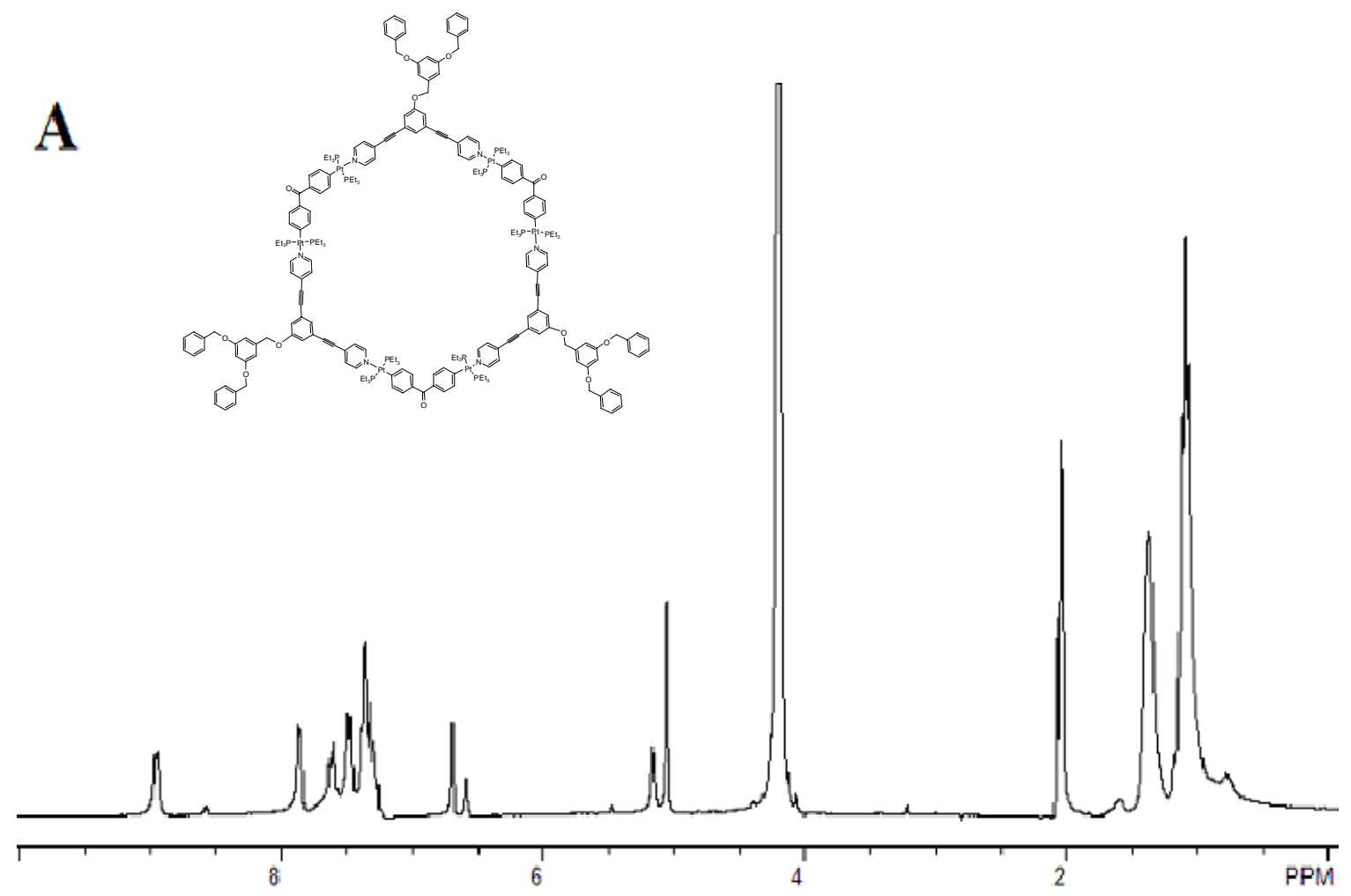

B

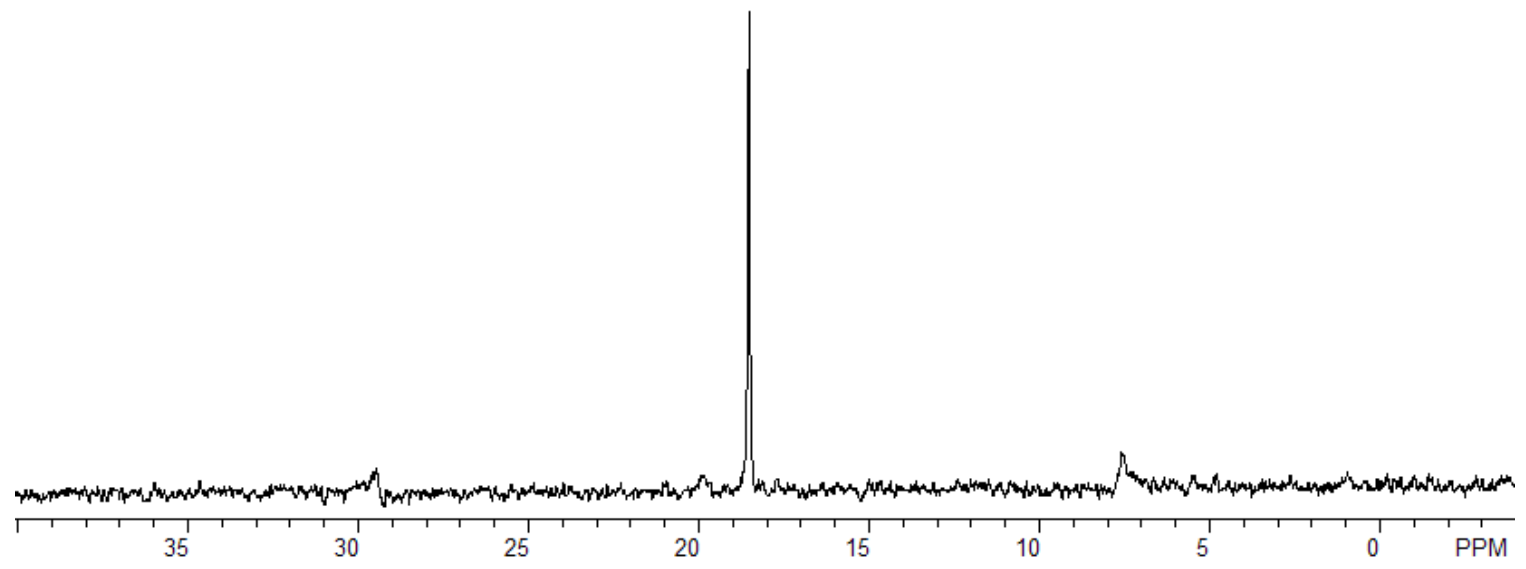


Figure S3. A) ' $\mathrm{H}$ and B) ${ }^{31} \mathrm{P}$ NMR spectra of $7 \mathbf{c}$ in Acetone- $d_{6} / \mathrm{D}_{2} \mathrm{O}(10 / 1)$

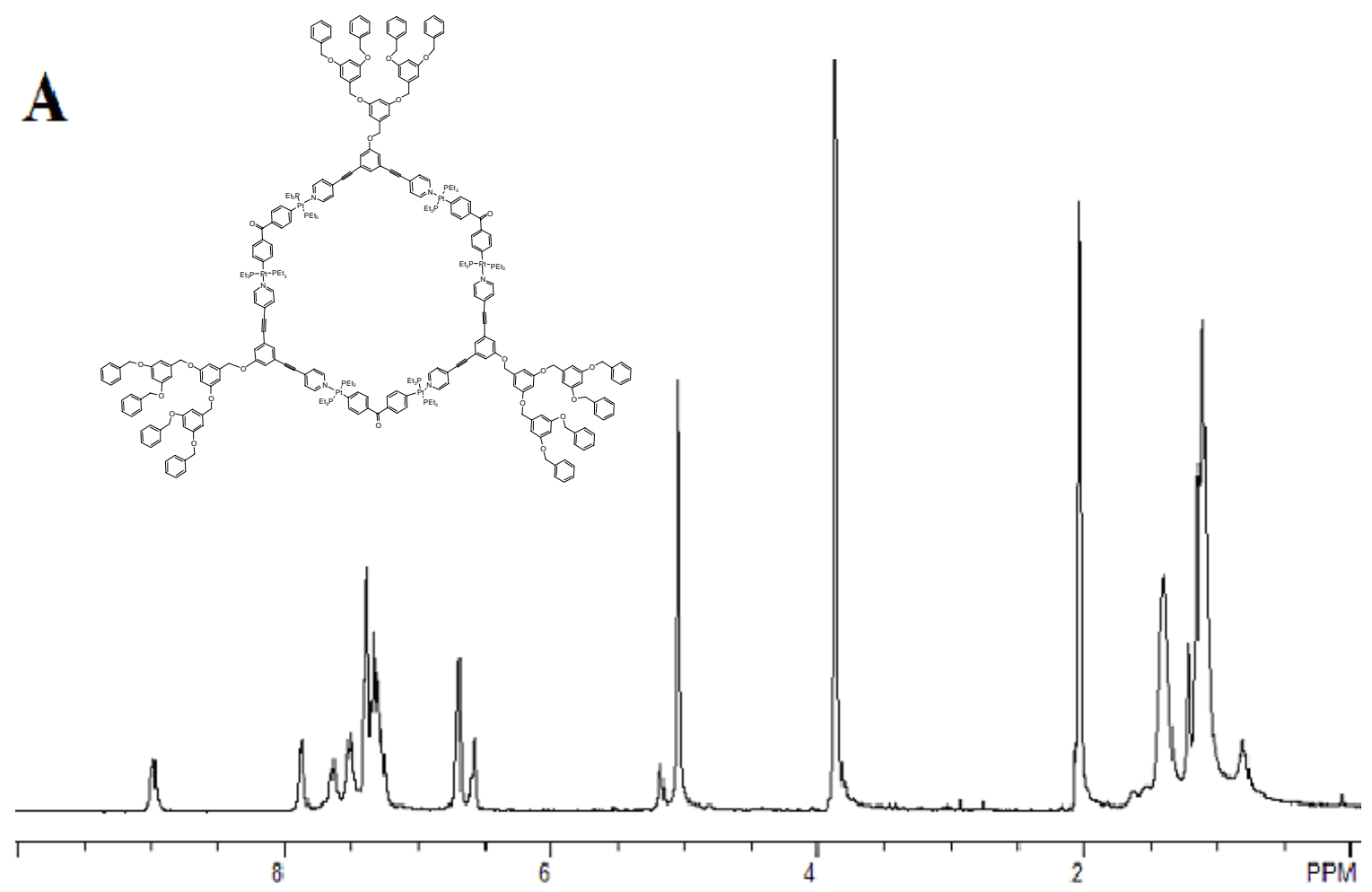

B

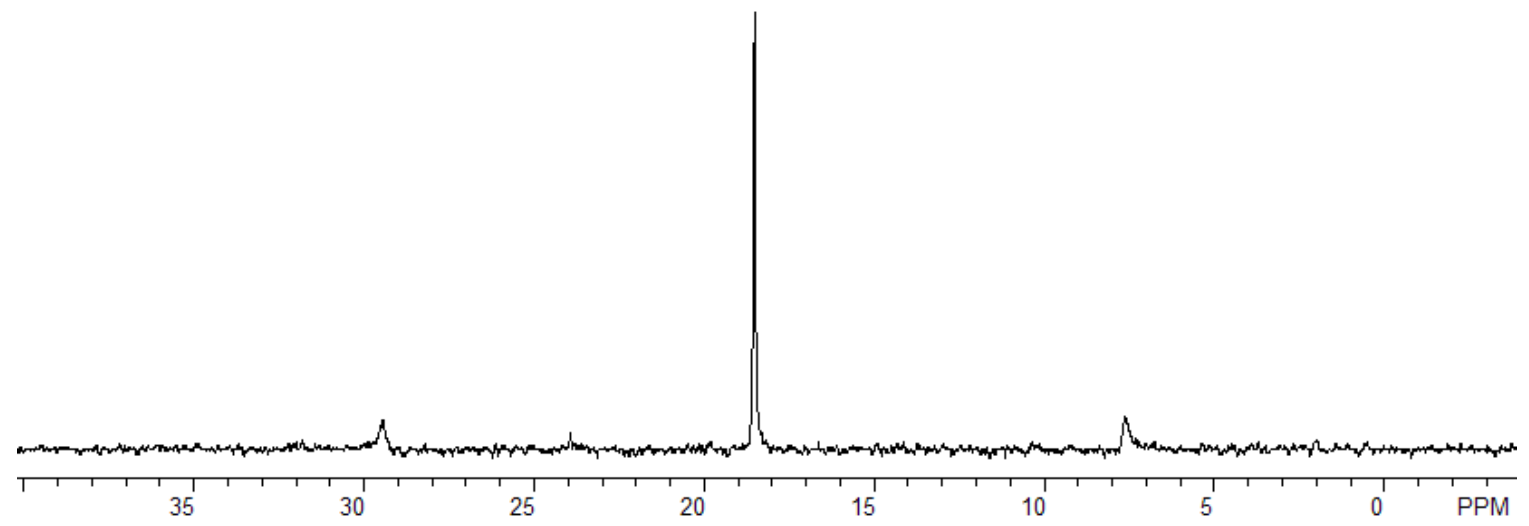


Figure S4. A) ' $\mathrm{H}$ and B) ${ }^{31} \mathrm{P}$ NMR spectra of $7 \mathbf{d}$ in Acetone- $d_{6} / \mathrm{D}_{2} \mathrm{O}(10 / 1)$

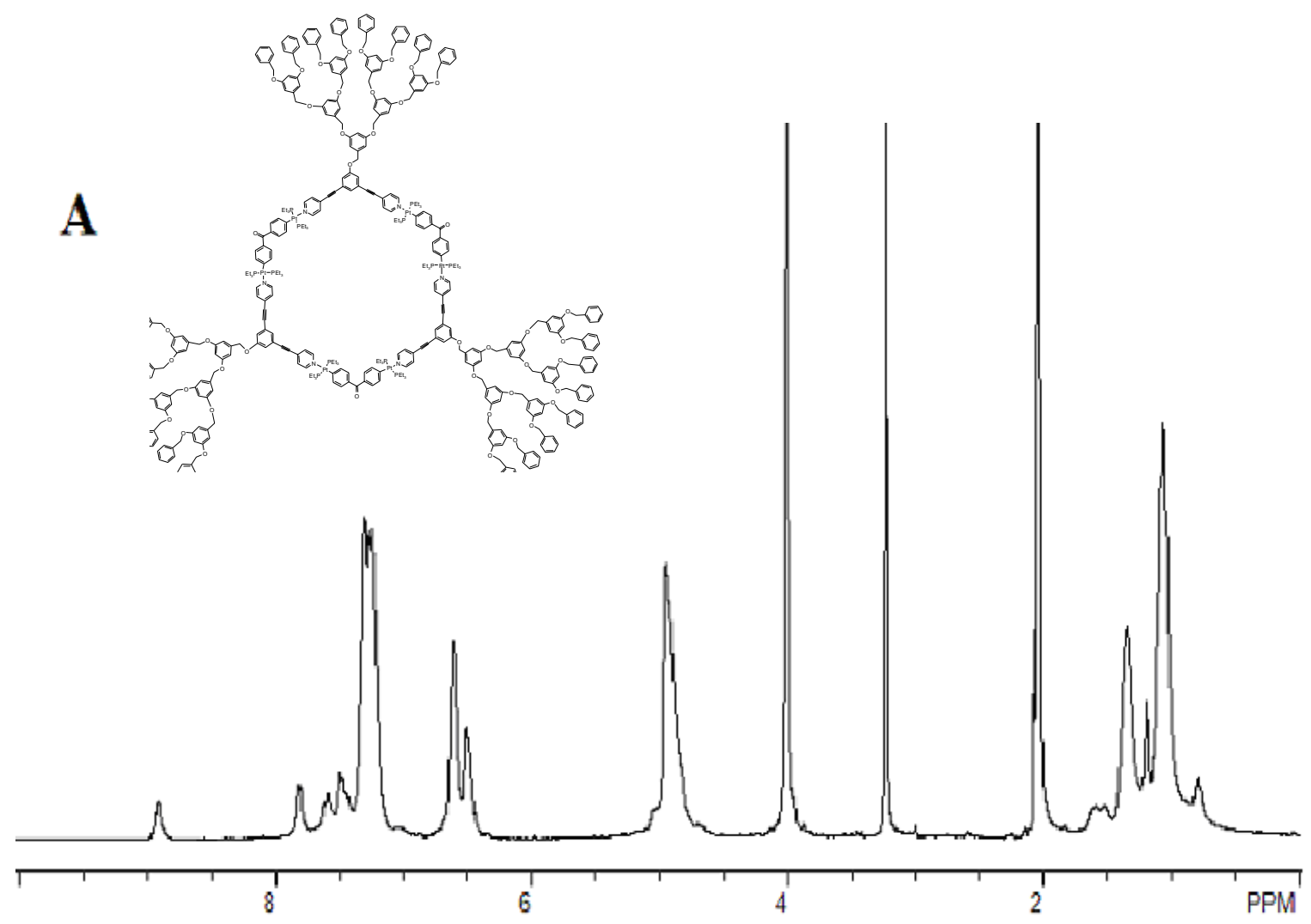

B

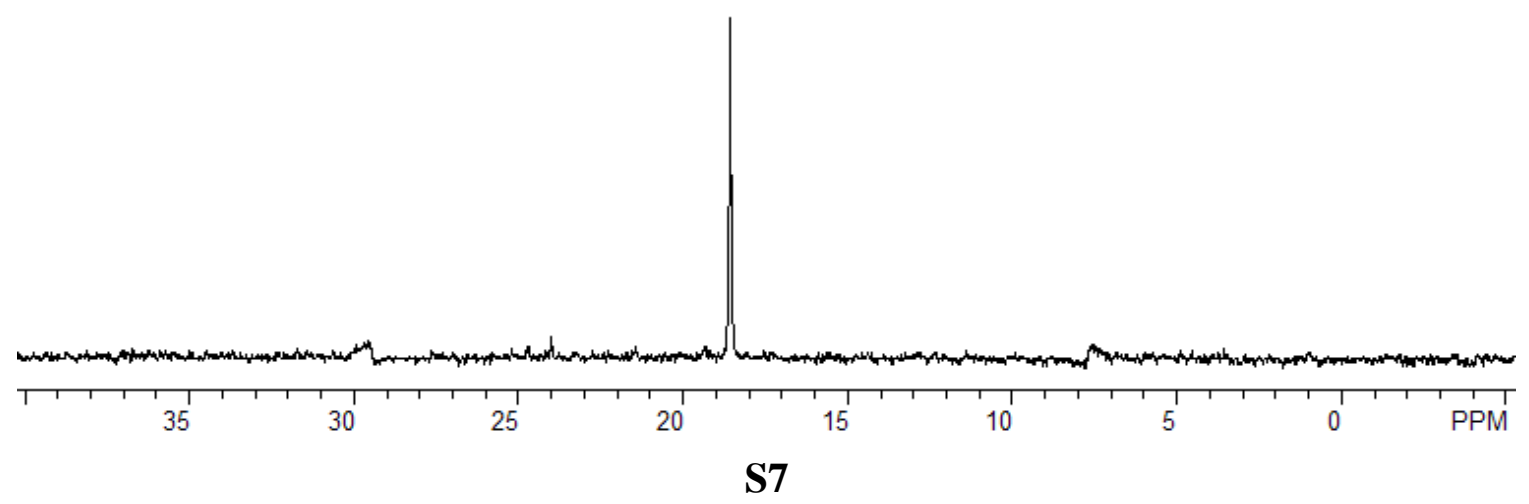


Figure S5. Electrospray Ionization MS of 7a. Insets are are isotopically resolved peaks.

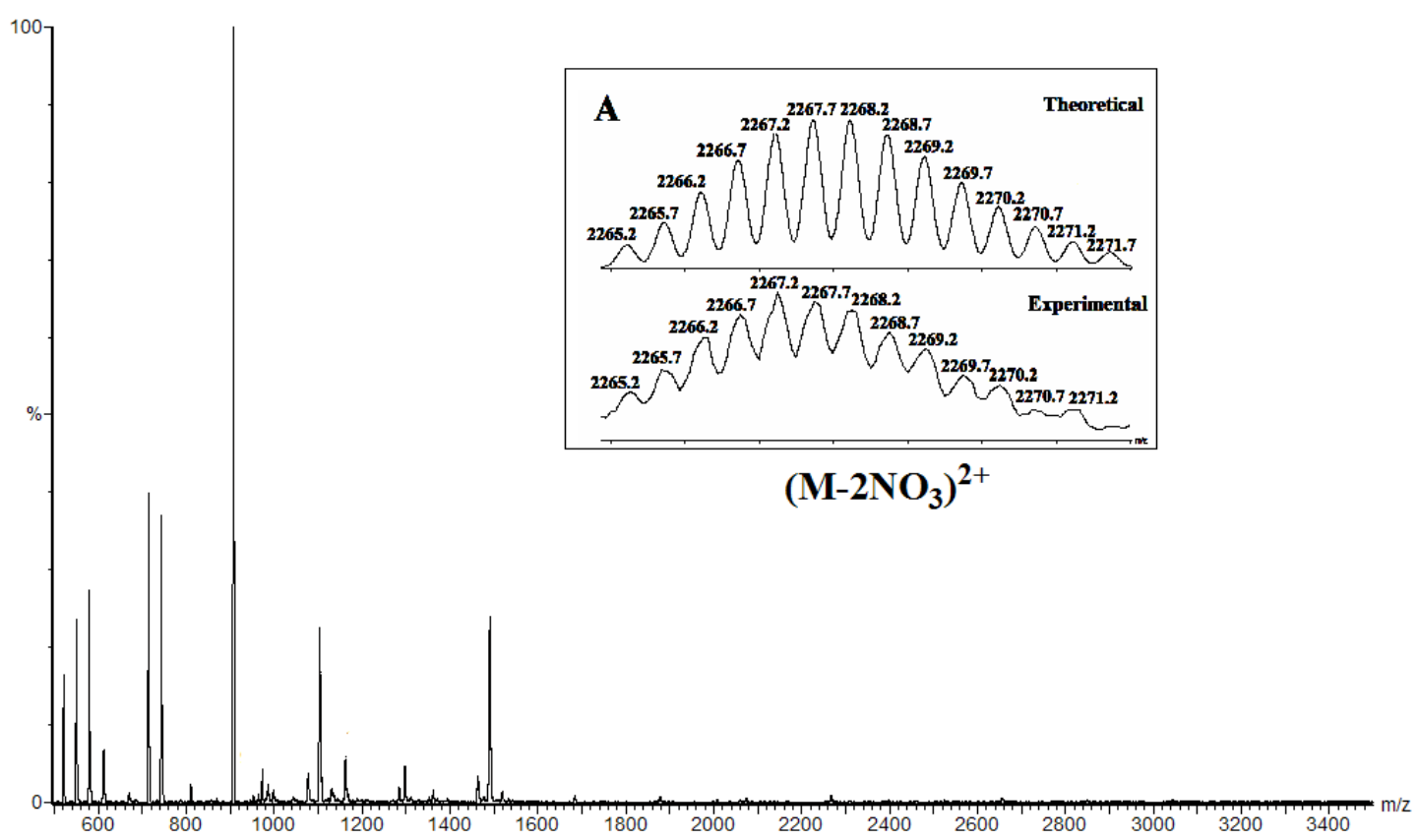

Figure S6. Electrospray Ionization MS of $\mathbf{7 b}$. Insets are are isotopically resolved peaks.

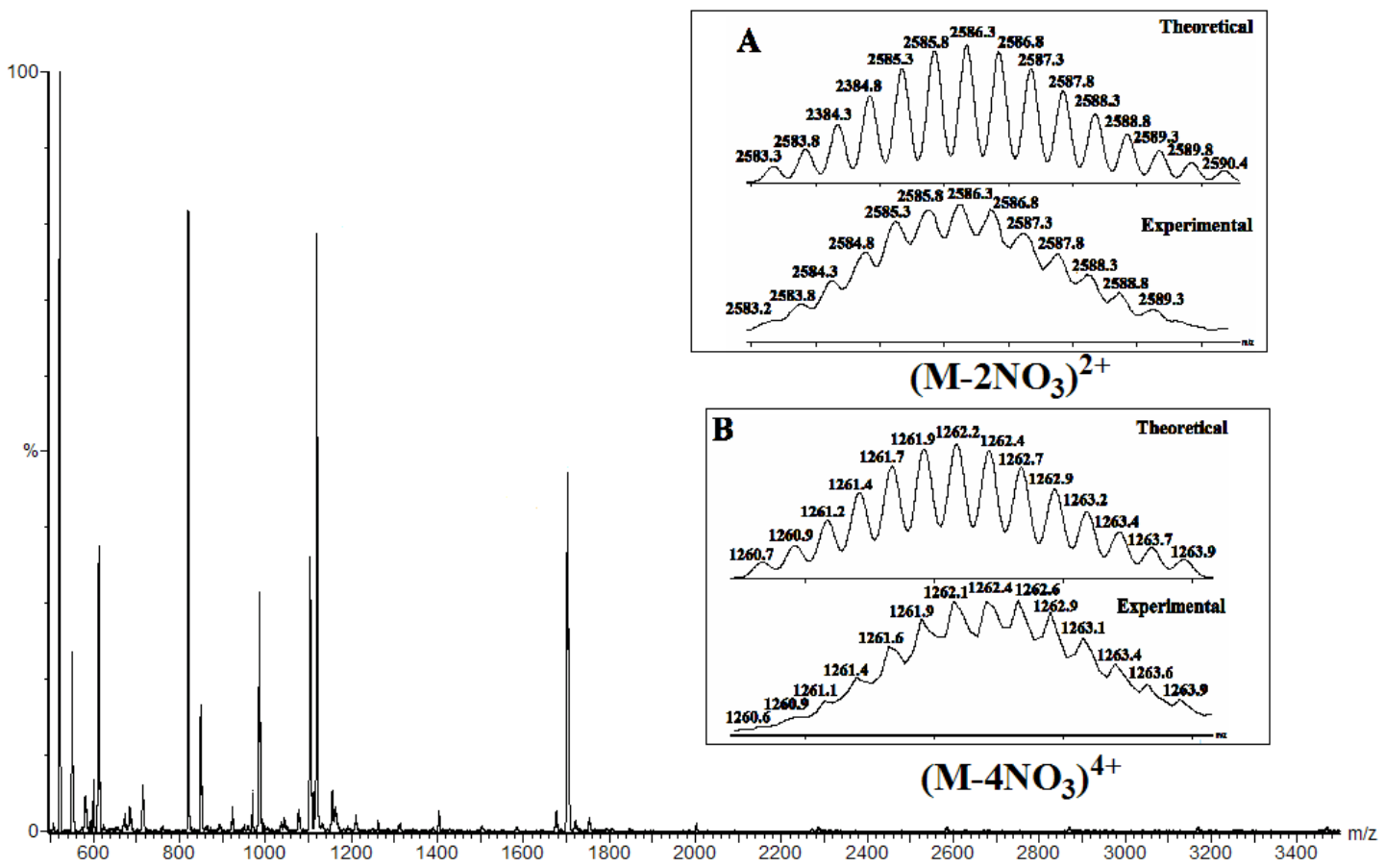


Figure S7. Electrospray Ionization MS of 7c. Insets are are isotopically resolved peaks.

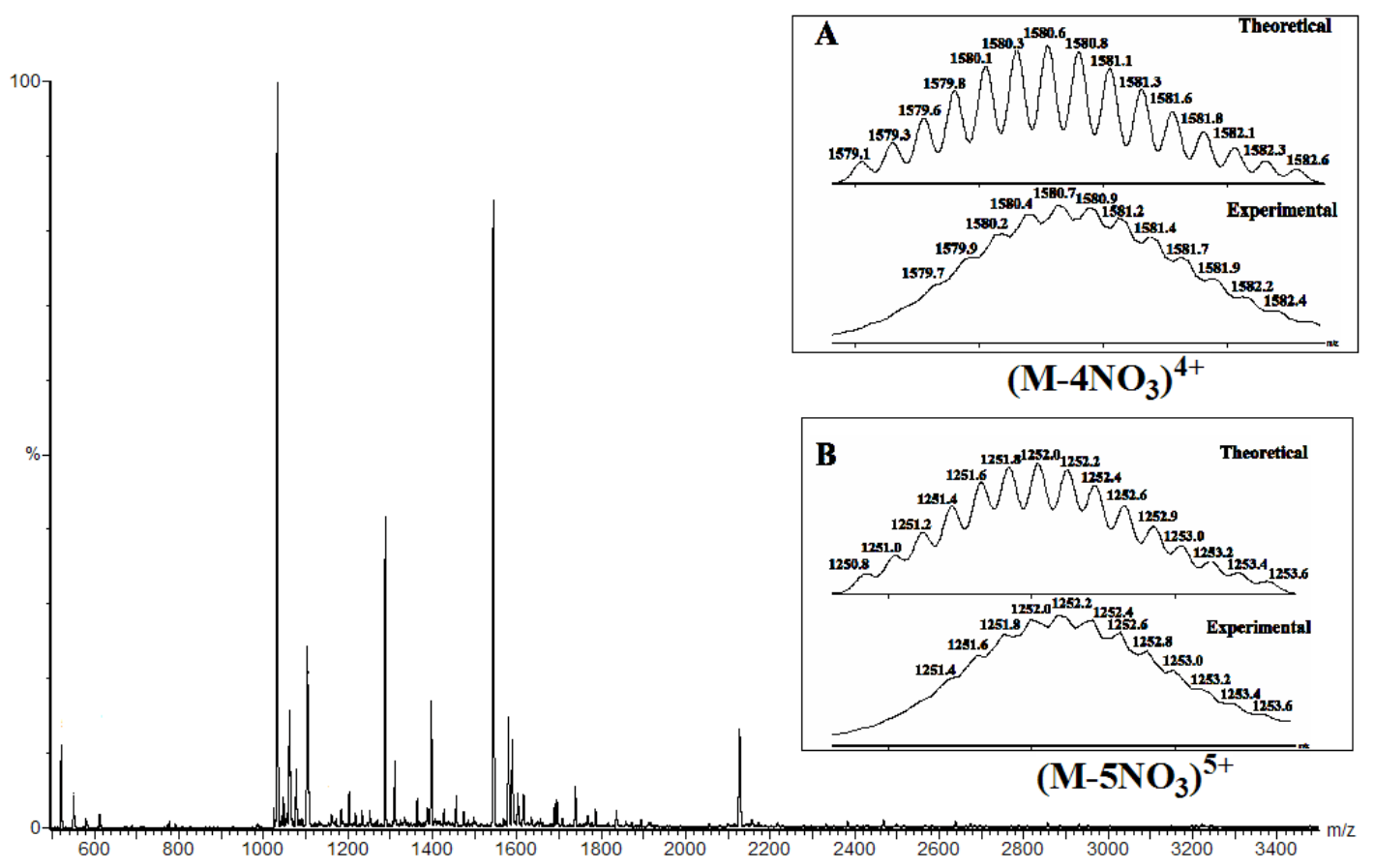

Figure S8. Electrospray Ionization MS of 7d. Insets are are isotopically resolved peaks.

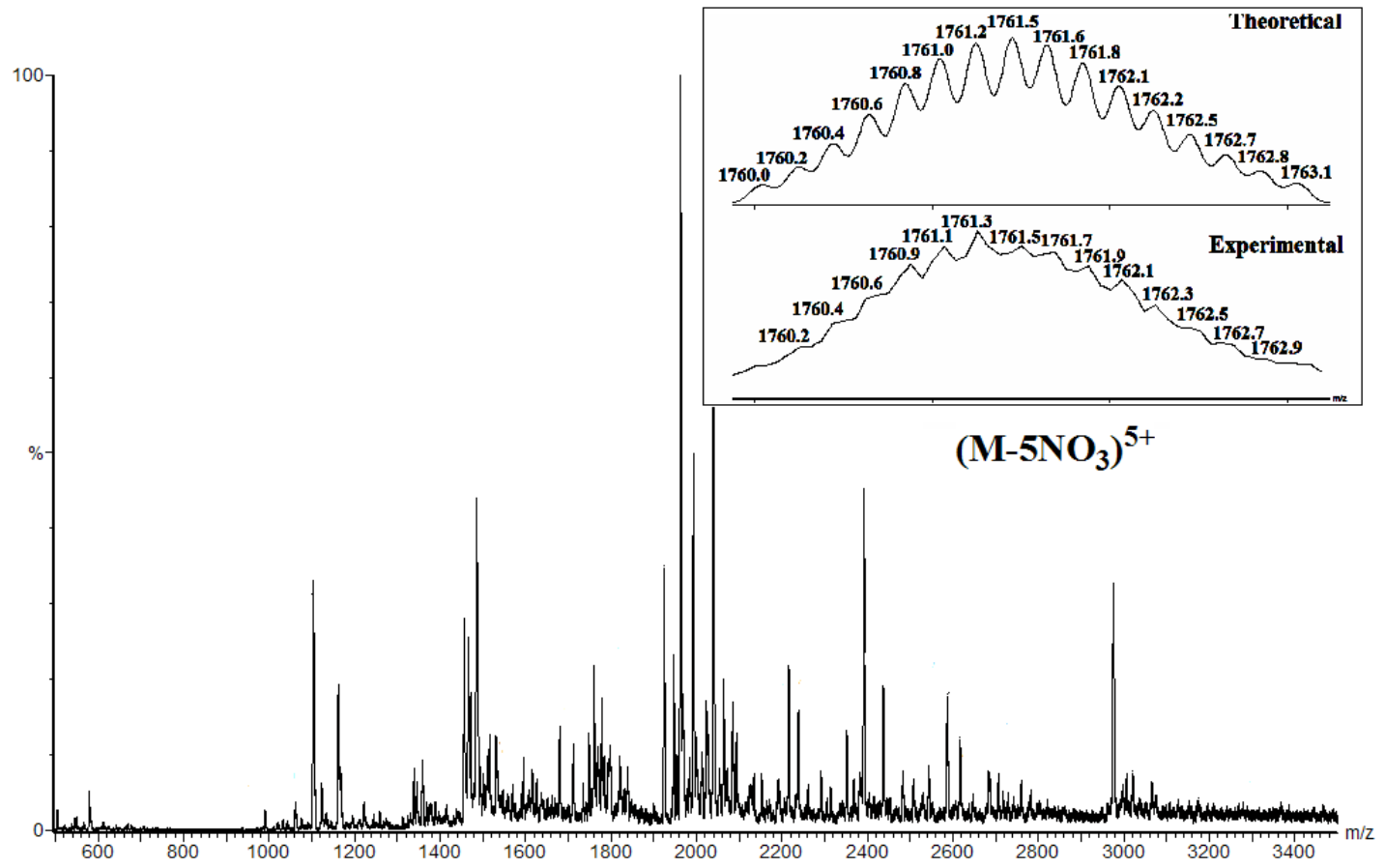


Figure S9. ESI-FT-ICR /MS of 7d. Insets are are isotopically resolved peaks.

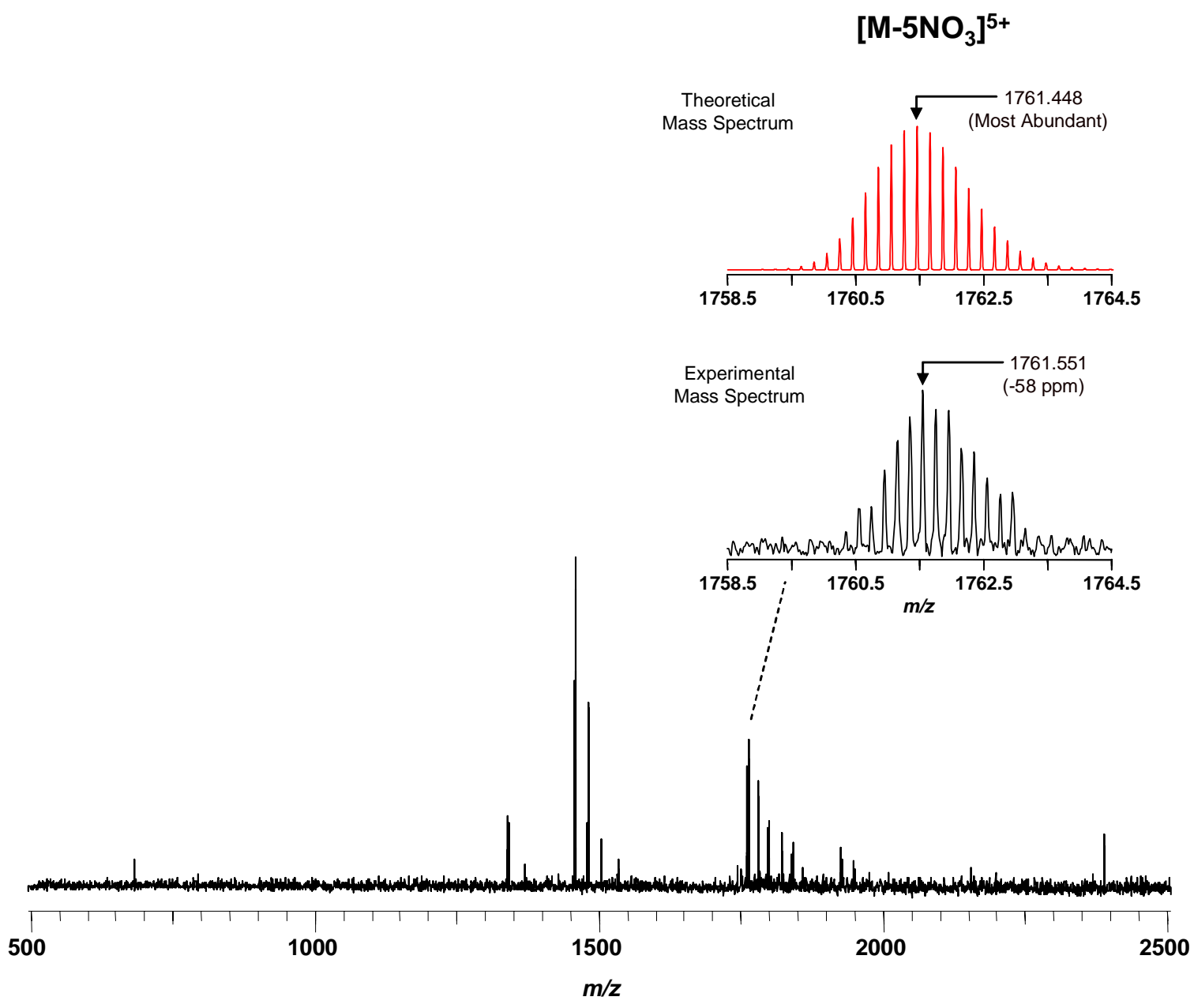

